\title{
The forkhead transcription factor FOXM1 promotes endocrine resistance and invasiveness in estrogen receptor-positive breast cancer by expansion of stem-like cancer cells
}

\author{
Anna Bergamaschi', Zeynep Madak-Erdogan', Yu Jin Kim², Yoon-La Choi ${ }^{2,3}$, Hailing Lu ${ }^{1,4}$ \\ and Benita S Katzenellenbogen ${ }^{1 *}$
}

\begin{abstract}
Introduction: The forkhead transcription factor FOXM1 coordinates expression of cell cycle-related genes and plays a pivotal role in tumorigenesis and cancer progression. We previously showed that FOXM1 acts downstream of 14-3-3 3 signaling, the elevation of which correlates with a more aggressive tumor phenotype. However, the role that FOXM1 might play in engendering resistance to endocrine treatments in estrogen receptor-positive (ER+) patients when tumor FOXM1 is high has not been clearly defined yet.
\end{abstract}

Methods: We analyzed FOXM1 protein expression by immunohistochemistry in 501 ER-positive breast cancers. We also mapped genome-wide FOXM1, extracellular signal-regulated kinase 2 and ERa binding events by chromatin immunoprecipitation followed by high-throughput sequencing (ChIP-seq) in hormone-sensitive and resistant breast cancer cells after tamoxifen treatment. These binding profiles were integrated with gene expression data derived from cells before and after FOXM1 knockdown to highlight specific FOXM1 transcriptional networks. We also modulated the levels of FOXM1 and newly discovered FOXM1-regulated genes and examined their impact on the cancer stem-like cell population and on cell invasiveness and resistance to endocrine treatments.

Results: FOXM1 protein expression was high in $20 \%$ of the tumors, which correlated with significantly reduced survival in these patients ( $P=0.003$ by logrank Mantel-Cox test). ChIP-seq analyses revealed that FOXM1 binding sites were enriched at the transcription start site of genes involved in cell-cycle progression, maintenance of stem cell properties, and invasion and metastasis, all of which are associated with a poor prognosis in ERa-positive patients treated with tamoxifen. Integration of binding profiles with gene expression highlighted FOXM1 transcriptional networks controlling cell proliferation, stem cell properties, invasion and metastasis. Increased expression of FOXM1 was associated with an expansion of the cancer stem-like cell population and with increased cell invasiveness and resistance to endocrine treatments. Use of a selective FOXM1 inhibitor proved very effective in restoring endocrine therapy sensitivity and decreasing breast cancer aggressiveness.

Conclusions: Collectively, our findings uncover novel roles for FOXM1 and FOXM1-regulated genes in promoting cancer stem-like cell properties and therapy resistance. They highlight the relevance of FOXM1 as a therapeutic target to be considered for reducing invasiveness and enhancing breast cancer response to endocrine treatments.

\footnotetext{
* Correspondence: katzenel@illinois.edu

1 Department of Molecular and Integrative Physiology, University of Illinois at Urbana-Champaign, 524 Burrill Hall, Urbana, IL 61801, USA

Full list of author information is available at the end of the article
} 


\section{Introduction}

Endocrine resistance in breast cancer is a process that appears to result from upregulation of growth factor and protein kinase signaling pathways that provide an alternate mechanism in support of tumor cell proliferation and survival [1-4]. Tamoxifen (TAM) has proven to be one of the most successful agents in the management of estrogen receptor-positive (ER+) breast cancers. When effective, it suppresses tumor growth and reduces the risk of relapse. Unfortunately, with time, about $50 \%$ of patients with ER+ breast cancer stop benefiting from TAM treatment and acquire resistance, leading to disease progression. Despite significant advances in defining some of the factors involved [5-8], the mechanisms underlying endocrine resistance are complex and not fully understood. Therefore, we have been interested in identifying and targeting, by inhibition or downregulation, key players that mediate endocrine resistance in ER+ breast cancer.

Many cancers are maintained in a hierarchical organization of rare cancer stem cells (CSCs) and more plentiful differentiated tumor cells. CSCs that are resistant to treatment not only have the capacity to give rise to differentiated tumor cells but also can lead to recurrence, metastasis and disease progression [9-11]. Therefore, endocrine resistance might be associated with the outgrowth of CSCs by promoting expansion of the CSC population or augmenting the production of key factors that regulate the CSC phenotype.

In our previous studies, we reported a correlation between overexpression of the protein 14-3-3 $\zeta$ and early onset of recurrence in breast cancer patients [12]. We also uncovered a previously unknown relationship between 143-3 $\zeta$ and FOXM1 in TAM resistance in breast cancer, with $14-3-3 \zeta$ acting upstream of FOXM1 to enhance the expression of FOXM1-regulated genes [13].

FOXM1 is a forkhead transcription factor that binds to chromatin and plays an important role in ER $\alpha$ signaling pathways [14]. FOXM1 is a key regulator of the cell cycle and is essential for formation of the mitotic spindle and correct chromosome segregation [15]. Its expression is very low in normal tissues, but elevated in many types of cancers [16-18]. High expression of FOXM1 is associated with a poor prognosis [19-22]. In addition to its role in mitosis and cytokinesis, this transcription factor regulates genes that control critical aspects of cancer, including differentiation [23], angiogenesis [24] and metastasis $[16,20]$.

In this study, we show that TAM-resistant (TamR) cells contain higher levels of FOXM1 than do parental cells sensitive to growth inhibition by TAM and that this is correlated with the presence of a larger CSC population. Further, in large cohorts of patient breast tumors that we examined, high FOXM1 RNA and protein levels were found to correlate with a significantly faster onset of tumor recurrence and reduced overall survival. In cultured cells, FOXM1 promoted breast cancer aggressiveness and therapy resistance which could be reversed by FOXM1 inhibition or knockdown. Our genome-wide analyses using chromatin immunoprecipitation followed by high-throughput sequencing (ChIP-seq) revealed that TAM-specific FOXM1 binding sites are associated with genes encoding markers of CSCs and invasiveness and that overexpression of FOXM1 increases the proportion of CSCs and directly regulates the production of factors that promote aggressiveness and therapy resistance in breast cancer.

\section{Methods}

\section{Cell culture, small interfering RNA, overexpression and} ligand treatments

MCF-7 and T47D cells were obtained from the American Type Culture Collection (Manassas, VA, USA) and TamR MCF-7 cells (TamR cells) described previously [25] were cultured in minimal essential medium (MEM; Sigma-Aldrich, St Louis, MO, USA) supplemented with $5 \%$ calf serum (HyClone Laboratories, Logan, UT, USA), $100 \mu \mathrm{g} / \mathrm{ml}$ penicillin-streptomycin (Invitrogen, Carlsbad, $\mathrm{CA}, \mathrm{USA}$ ) and $25 \mu \mathrm{g} / \mathrm{ml}$ gentamicin (Invitrogen). Four days before control vehicle or ligand treatment, cells were seeded in phenol red-free MEM containing 5\% charcoaldextran-treated calf serum. Medium was changed on days 2 and 4 of culture before treatment. For three-dimensional cultures, $100 \mu \mathrm{l}$ of Matrigel was spread in each well of a 12-well plate, and 8,000 cells were seeded and grown for 6 to 10 days. Spheroids were stained with Giemsa-Wright stain for 15 minutes at room temperature and washed twice with $1 \times$ phosphate-buffered saline (PBS) for $5 \mathrm{mi}-$ nutes each. Small interfering RNA (siRNA) experiments were carried out by transfecting $50 \mathrm{nM}$ of siCtrl, siFOXM1 or siABCG2 from DharmaFECT reagent (Dharmacon, Lafayette, CO, USA) for 72 hours. Overexpression was performed as previously reported [12].

\section{ChIP and ChIP-reChIP assays}

Cells were treated with $0.1 \% \mathrm{EtOH}$ (vehicle) or $1 \mu \mathrm{M} 4$ hydroxytamoxifen (4-OH-TAM) for 45 minutes after pretreatment for 1 hour with the FOXM1-selective alternate reading frame (ARF) peptide inhibitor or mutant ARF control peptide [26] or with extracellular signal-regulated kinase kinase 1 (MEK1) inhibitor (AZD6244; Sellek Chemical, Houston, TX, USA) or control vehicle. After treatment, chromatin was cross-linked using $1 \%$ formaldehyde for 15 minutes at room temperature. Cells were washed with PBS, harvested and sonicated three times for 10 seconds in ChIP lysis buffer. Lysates were centrifuged for 10 minutes at $4^{\circ} \mathrm{C}$. For immunoprecipitation of DNAprotein complexes, lysates were incubated overnight with antibodies to FOXM1 (GeneTex, Irvine, CA, USA) or extracellular signal-regulated kinase 2 (ERK2; Santa Cruz 
Biotechnology, Santa Cruz, CA, USA). Complexes were washed three times with radioimmunoprecipitation assay (RIPA) buffer (three times) and two times with Tris-EDTA (ethylenediaminetetraacetic acid). Following the overnight incubation at $65^{\circ} \mathrm{C}$, ChIP DNA was isolated using a QIAGEN PCR purification kit (QIAGEN, Valencia, CA, USA) as per the manufacturer's suggestions. The DNA was used for ChIP-seq analysis and quantitative real-time PCR.

Sequential chromatin immunoprecipitation (ChIPreChIP) experiments were done following the same ChIP protocol. After the first pull-down, immunoprecipitated material was recovered with $10 \mathrm{mM}$ dithiothreitol in immunoprecipitation buffer at $37^{\circ} \mathrm{C}$ for 30 minutes, diluted and subjected to a second round of immunoprecipitation. Quantitative RT-PCR (qRT-PCR) was used to calculate recruitment to the regions studied, as described elsewhere [27].

\section{ChIP-seq and clustering analysis}

For characterization of the FOXM1 and ERK2 cistromes from cells treated with 4-OH-TAM, the ChIP DNA was prepared into libraries according to Illumina Solexa ChIP-seq sample-processing methods (San Diego, CA, USA), and single-read sequencing was performed using the Illumina Solexa Genomic Analyzer using methods detailed previously [28]. Sequences generated were mapped uniquely onto the human genome (hg19) by Bowtie2 [29] with the default settings. A model-based analysis of ChIPSeq algorithm [30] was used to identify enriched peak regions (default settings) with a $P$-value cutoff of $6.0 \mathrm{E}-7$ and false discovery rate of 0.01. ChIP-seq data for FOXM1 and ERK2 binding sites are given as BED files in Additional file 1: Table S1. Cistrome data for ER $\alpha$ in MCF-7 cells treated with Tam are derived from a previous study [31].

The seqMINER density array method with a 300-bp window in both directions was used for the generation of clusters (that is, groups of loci having similar compositional features) [32]. This ChIP-seq data interpretation platform allows the comparison and integration of multiple ChIP-seq data sets and their extraction and visualization of specific patterns as described previously [28]. BED files for each cluster were used for further analysis with Galaxy Cistrome integrative analysis tools (Venn diagram, conservation, Cis-regulatory Element Annotation System (CEAS)) [33].

\section{Motif and Gene Ontology category analysis}

Overrepresented Gene Ontology (GO) biological processes were determined by utilizing the web-based DAVID Bioinformatics Resources database [34,35], GeneSpring and web-based GREAT (Genomic Regions Enrichment of Annotations Tool) software [36]. Motif enrichment analysis was done using SeqPos [33]. Conservation of the binding sites was determined using web-based CEAS software of the Cistrome/Galaxy platform [37]. Default parameters were used in all software.

\section{RT-PCR and quantitative PCR}

Total RNA was isolated from cells using TRIzol reagent (Invitrogen). RNA samples were reverse-transcribed using SuperScript II reverse transcriptase (Invitrogen), and RT-PCR was carried out on the ABI Prism 7900HT Sequence Detection System using SYBR Green PCR Master Mix (Applied Biosystems, Foster City, CA, USA) as described previously [38]. Primer sequences for the genes studied were obtained from the Harvard Primer Bank [39]. Sequences are available on their website.

\section{Microarray gene expression data analysis and statistics}

Total RNA was used to generate complementary RNA (cRNA), which was labeled with biotin according to protocols recommended by Affymetrix (Santa Clara, CA, USA). All analyses were done using three or more samples for each treatment. The biotin-labeled cRNA was hybridized to Affymetrix U133 plus 2.0 GeneChips, which contain oligonucleotide probe sets for over 47,000 transcripts. After being washed, the chips were scanned and analyzed using Affymetrix processing software. All microarray gene expression data have been deposited in the Gene Expression Omnibus database [GEO:GSE55204]. CEL files were processed using GeneSpring GX 11.0 software (Agilent Technologies, Santa Clara, CA, USA) to obtain fold changes and $P$-values with the Benjamini and Hochberg multiple-test correction [40] for each gene for TAM treatment relative to the vehicle control in each cell background. We considered genes with fold changes greater than two and $P$-values $<0.05$ as statistically significant and differentially expressed. For analyses of microarray data sets from TAM- treated breast cancer patients, we used Frasor et al. data [GEO:GSE1379] [38] and Buffa et al. data [GEO:GSE2221] [41]. Multifactor analysis was computed in WinSTAT statistics add-in for Excel software (R. Fitch software). Differences between two groups were assessed using an unpaired t-test. Data involving more than two groups were assessed by analysis of variance with Dunnett's multiple-comparisons test. Differences were considered significant at $P<0.05$. Additional statistical analyses done are indicated in the figure legends.

\section{Western blot analysis}

Whole-cell extracts were prepared using $1 \times$ RIPA lysis buffer (Upstate/Chemicon, Billerica, MA, USA) supplemented with $1 \times$ cOmplete Protease Inhibitor Cocktail mixture (Roche Applied Science, Basel, Switzerland). Proteins were separated on $4 \%$ to $20 \%$ gradient SDS-PAGE gels and transferred to nitrocellulose membranes. For Western blot analysis, we used antibodies against FOXM1, ERK1 and ERK2 (Santa Cruz Biotechnology), $\beta$-actin (Sigma-Aldrich), 
phosphorylated mitogen-activated protein kinase (pMAPK) (Cell Signaling Technology, Danvers, MA, USA) and CD44 (BD Biosciences, San Jose, CA, USA).

\section{Cell proliferation assay}

A WST-1 assay (Roche Applied Science) was used to quantify cell viability. Absorbance was read at $450 \mathrm{~nm}$ on a PerkinElmer Victor X Multilabel Plate Reader (PerkinElmer, Waltham, MA, USA), and all assays were performed in triplicate as described elsewhere [13,42].

\section{Fluorescence-activated cell sorting and immunofluorescence}

For fluorescence-activated cell sorting (FACS), cells were detached and then stained with antibodies for CD44, CD24, ABCG2 (BD Biosciences and Cell Signaling Technology) at 1:100 dilution in PBS containing $1 \%$ fetal calf serum. FACS-sorted cells were collected into cell medium for plating or into RNAlater ${ }^{\text {TM }}$ buffer for RNA extraction. To test for ABCG2+ activity, $1 \times 10^{6}$ cells were incubated with $5 \mu \mathrm{M}$ Hoechst 33258 dye at $37^{\circ} \mathrm{C}$ for 90 minutes. All samples were analyzed and sorted using a FACSAria III instrument (BD Biosciences).

\section{Invasion assay}

Breast cancer cells were seeded on precoated filters $(8-\mu \mathrm{m}$ pore size) after membrane rehydration (BD Biosciences). Following incubation for 48 hours at $37^{\circ} \mathrm{C}$, cells were fixed in $10 \%$ formalin buffer and stained using crystal violet. Noninvasive cells on the surface of the filter were removed using a cotton swab. Invasion was quantified by determining the percentage of cells that had invaded the filter compared to the total number seeded as described previously $[13,42]$.

\section{Breast tumor cohort and FOXM1 immunohistochemistry and statistical analysis}

A tissue microarray (TMA) from the Samsung Medical Center Breast Cancer Biomarker Study was utilized for the analysis of FOXM1 status. Detailed clinical features and molecular subtype classification have been reported elsewhere [43,44]. Briefly, from among 815 tumors, 501 were assigned as ER $\alpha$-positive and used for the immunohistochemical detection of FOXM1 expression. TMA sections were incubated for 1 hour at room temperature with mouse anti-human FOXM1 antibody (ab55006; Abcam, Cambridge, MA, USA) diluted 1:400. The detection system EnVision+ for mouse antibody (K4001; Dako, Glostrup, Denmark) was applied according to the manufacturer's instructions. Slides were stained with liquid diaminobenzidine tetrahydrochloride (DAB+), a high-sensitivity substrate chromogen system (K3468; Dako). Counterstaining was performed with Mayer's hematoxylin. FOXM1 expression was scored using a semiquantitative method based on the following four classes: score 0 (no staining or nuclei staining observed in $<10 \%$ of the tumor cells), score $1+$ (faint nuclear staining detectable in $>10 \%$ of the tumor cells), score $2+$ (weak to moderate nuclear staining observed in $>10 \%$ of the tumor cells) and score $3+$ (strong nuclear staining observed in $>30 \%$ of the tumor cells). Representative photomicrographs of each of the scoring categories are shown in Additional file 2: Figure S4. Patients with tumor scores ranging from 0 to 1 were classified as FOXM1-negative/low expression, and those who had scores of 2+ and 3+ were classified as FOXM1-high expression group. Disease-free survival was defined as the time from the date of diagnosis to the date of documented relapse, including locoregional recurrence and distant metastasis. Survival curves were constructed using the KaplanMeier method, and the logrank test was used to compare the mean survival rates across the groups. The logrank test with Bonferroni's correction was used for the subgroup survival analysis.

\section{Accession numbers and data availability}

Gene expression data are available in the GEO database [GEO:GSE55204]. ChIP-Seq data files for FOXM1 and ERK2 binding sites in TAM-treated cells are given as BED files in Additional file 1: Table S1.

\section{Results}

\section{Estrogen receptor-positive breast tumors with high} expression of FOXM1 show early time to recurrence, and tumors positive for both 14-3-3 and FOXM1 show earliest time to recurrence

We reported previously that women with breast tumors expressing high levels of the scaffold adaptor protein 14$3-3 \zeta$ had a poor prognosis $[12,13]$. We also observed by molecular analyses that FOXM1 was regulated by 14-3-3 $\zeta$

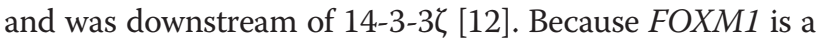
transcription factor that might regulate the expression of genes that engender this less good patient outcome, we first investigated the relationship between FOXM1 and 14-3-3 $\zeta$. As shown in Figure 1A, we examined the mRNA expression of 27 forkhead transcription factors in 251 primary ER $\alpha$-positive breast tumors. Notably, we observed that expression of $14-3-3 \zeta$ in these tumors was most highly correlated with expression of FOXM1 $(r=0.59, P=$ 9.03E-13) and next with FOXK2 expression $(r=0.38, P=$ 2.07E-10) (Figure 1A). This good correlation in expression

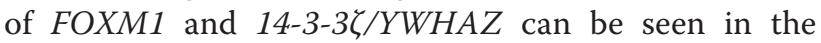
gene expression heat map and in the factor analysis plot (Figure 1A). Furthermore, tumors positive for both 14-3-3 $\zeta$ and FOXM1 showed the earliest time to recurrence $(P=0.041)$ (Figure $1 \mathrm{~B})$. Of interest, analysis of our microarray gene expression data from a large study with TAM-treated breast cancer patients [38] showed that high FOXM1 mRNA expression in tumors was associated with 


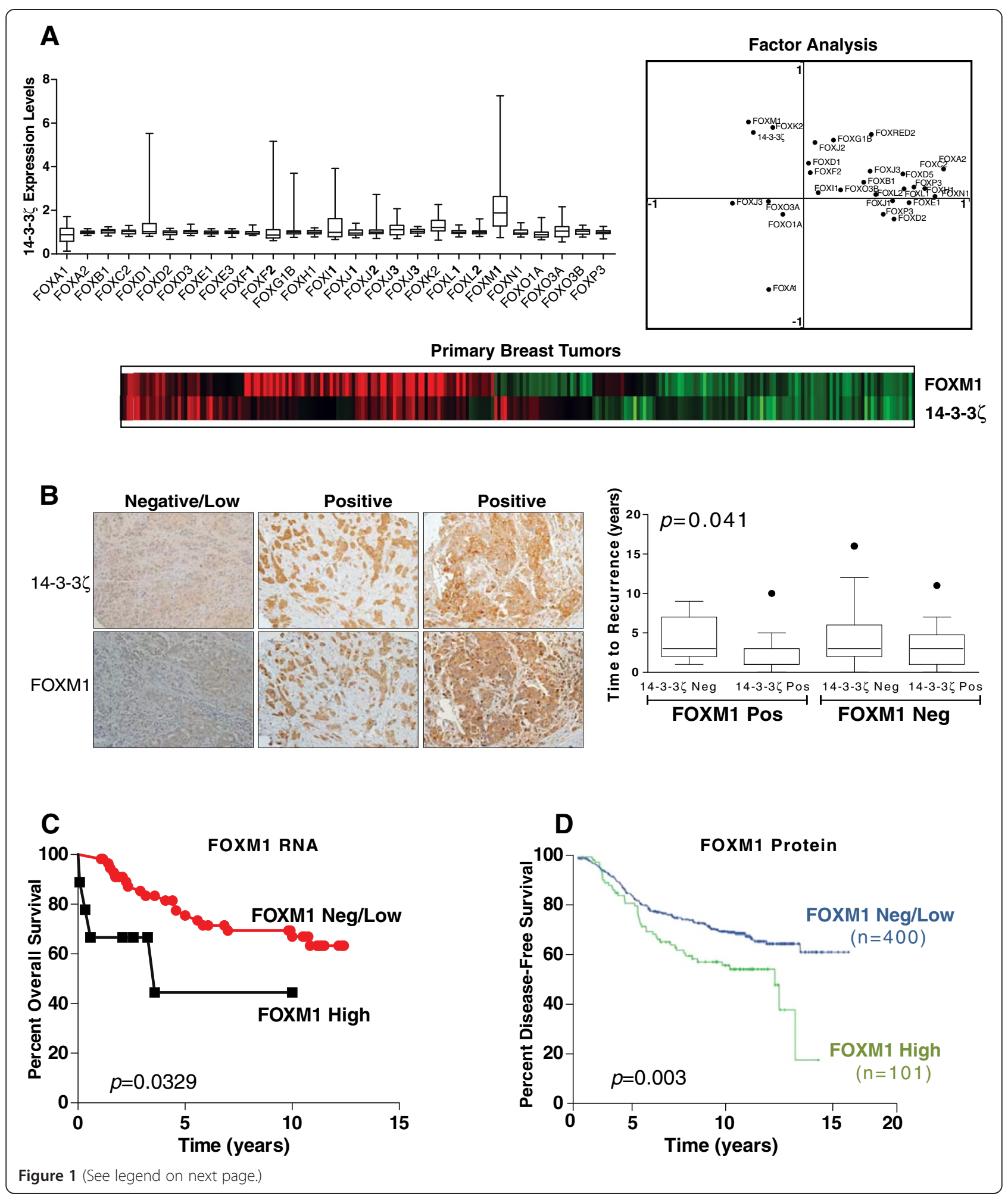




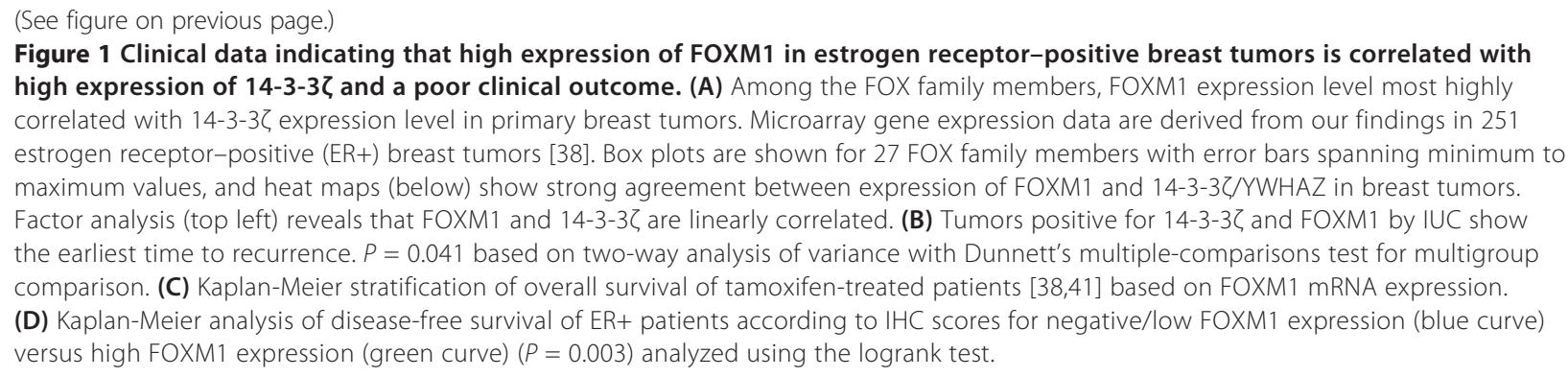

a less good patient survival (Figure 1C). Likewise, our immunohistochemistry (IHC) analysis of tumors from a cohort of $501 \mathrm{ER} \alpha$-positive breast tumors revealed that high expression of FOXM1 protein was associated with a much poorer patient survival (Figure 1D). Kaplan-Meier logrank survival analysis showed that time to recurrence was significantly longer in patients with tumors negative or low for FOXM1 compared to patients with tumors with high FOXM1 protein (IHC score 2 or 3 ), which represented about $20 \%$ of all ER+ tumors $(P=0.003)$ (Figure $1 \mathrm{D})$. FOXM1 multivariate Cox regression analysis also revealed FOXM1 to have a significant $P$-value $(P=0.0048$, odds ratio $=1.661,95 \%$ confidence interval $=1.177$ to 2.343 ) when FOXM1 was stratified by recurrence-free survival. Thus, high FOXM1 mRNA or protein confers a worse prognosis in ER+ breast cancers.

FOXM1 is elevated in tamoxifen-resistant breast cancer cells and contributes to the endocrine-resistant phenotype

On the basis of these clinical observations, we examined the levels of FOXM1 in MCF-7 parental and TamR cells and found threefold higher levels of FOXM1 protein in TamR cells (Figure 2A). We also monitored the kinetics of increase of FOXM1 over time of 4-OH-TAM exposure and observed a progressive and large (approximately tenfold) increase in FOXM1 mRNA over the course of 100 weeks examined (Figure 2B). Moreover, proliferation of control vehicle- or 4-OH-TAM treated cells was reduced by FOXM1 siRNA (Figure 2C), which resulted in almost full loss of FOXM1 protein. The TamR cells were growthstimulated by 4-OH-TAM (Figure 2C), and this stimulation was eliminated, and cell proliferation decreased, when FOXM1 was knocked down, indicating that FOXM1 plays a role in the resistance to TAM (Figure $2 \mathrm{C}$ ).

To assess how FOXM1 might affect gene regulation by 4-OH-TAM, we performed Affymetrix gene expression microarray analysis on MCF-7 cells treated with 4-OHTAM with (siFOXM1) and without (siCtrl) knockdown of FOXM1. Using a fold change greater than two and a $P$ value $<0.05$, we found 546 genes to be differentially expressed (Figure 2D). GO analysis of the functional annotations of the differentially regulated genes revealed an enrichment for cell cycle and chemotaxis categories in genes downregulated upon knockdown of FOXM1 in 4-OHTAM-treated cells vs. control, whereas apoptosis and programmed cell death genes were upregulated and enriched when FOXM1 was decreased by siFOXM1 treatment.

\section{Genome-wide analysis of FOXM1 chromatin binding by ChIP-seq, gene regulation by FOXM1 and clustering of} binding sites and delineation of gene functional categories

Next, we undertook the genome-wide characterization of FOXM1 binding sites by ChIP-seq analysis to address how TAM treatment affected the recruitment of FOXM1 to specific genomic loci. In our FOXM1 cistrome from MCF-7 cells treated with TAM, we observed that $22 \%$ of the FOXM1 binding sites were also shared by ER $\alpha$ binding sites after TAM treatment as reported by Hurtado et al. [31] (Figure 2E). Hence, FOXM1 also bound to a significant number of unique sites, suggesting that FOXM1 might uniquely control the transcription of specific sets of genes in a manner independent from ER $\alpha$ or that ER $\alpha$ might operate along with FOXM1 present at different sites via looping together of different chromatin locations.

A prominent feature of acquired TAM resistance is the hyperactivation of MAPK. In light of this and the fact that we have previously shown ERK2 to be recruited to chromatin by ER $\alpha$ after estradiol treatment of breast cancer cells [45], we assessed the recruitment of ERK2 to chromatin after 4-OH-TAM exposure of cells and the extent of overlap of ERK2 binding with FOXM1 and ER $\alpha$ binding (Figure 2E). ERK2 and FOXM1 co-occupied more sites (47\% of ERK2 sites) than ERK2 and ER $\alpha$ (35\% of ERK2 sites), suggesting that FOXM1 becomes a major transcription factor driving ERK2 to the chromatin in the presence of 4-OH-TAM.

To obtain a better picture of the chromatin binding landscape of these factors, we utilized a clustering approach using seqMINER [32], which compares the presence of multiple factors at a given chromosomal location within a 600-bp window and clusters together those binding sites that share a similar pattern of factor localization. We have previously shown, through this type of cluster analysis, that binding sites can be classified based on a series of factor recruitments, enabling the highlighting of commonalities in regulatory modes for modulated genes in each specific cluster [28]. 


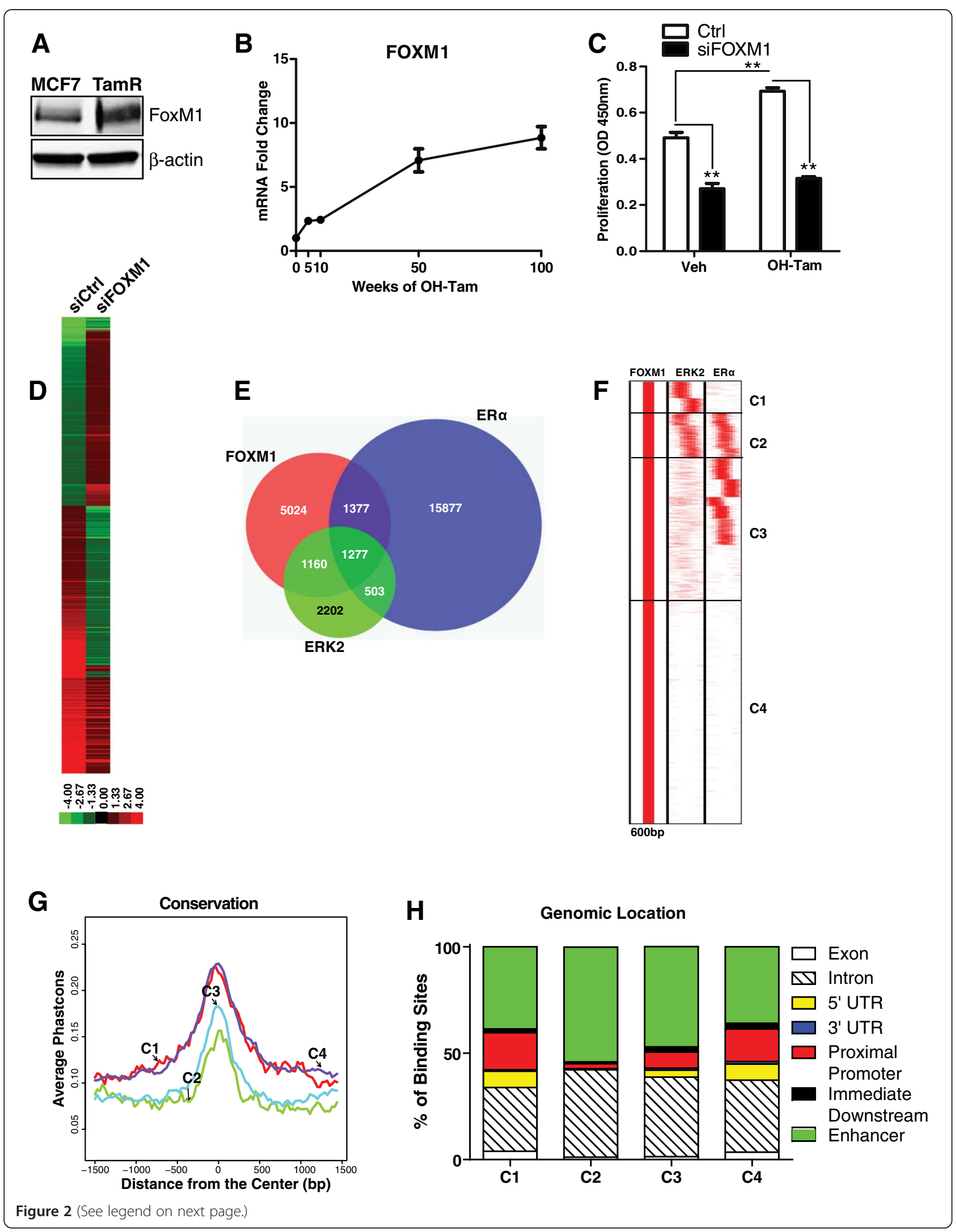


(See figure on previous page.)

Figure 2 FOXM1 is elevated by tamoxifen treatment and genome-wide analysis of FOXM1, ERK2 and ERa chromatin binding sites by ChIP-seq after tamoxifen treatment, and gene expression profiling, and clustering analyses. (A) FOXM1 protein levels in MCF-7 and tamoxifen-resistant (TamR) cells monitored by Western blot analysis. (B) mRNA levels of FOXM1 mRNA over time of 1 HM 4-hydroxytamoxifen (OH-TAM) exposure. The fold change in FOXM1 gene expression in the presence of tamoxifen- over vehicle-treated cells was calculated using the comparative threshold cycle method, with the ribosomal protein 36B4 mRNA used as an internal control. (C) Proliferation of TamR control cells (Ctrl) and cells with FOXM1 knockdown (siFOXM1). OD, Optical density. Data are mean \pm SEM. ${ }^{* *} P<0.01$. (D) Heat map representing the expression levels of $\mathrm{OH}-\mathrm{Tam}-$ regulated genes in Ctrl and siFOXM1 MCF-7 cells treated with control vehicle or OH-Tam. Heat map shows fold change for gene expression in Tam-treated vs. vehicle-treated cells with or without siFOXM1. (E) Venn diagram showing overlap of FOXM1, extracellular signal-regulated kinase 2 (ERK2) and estrogen receptor a (ERa) chromatin binding sites in cells after 45 minutes of OH-Tam treatment and chromatin immunoprecipitation followed by high-throughput sequencing (ChIP-seq) analysis. FOXM1-Tam and ERK2-Tam cistrome data are from this study; the ERa-Tam cistrome data are derived from Hurtado et al. [31]. (F) Clustering of the binding sites for FOXM1, ERK2 and ERa after cell treatment with Tam using segMINER software based on co-occupancy of the different factors within a 600-bp window. (G) Conservation of clusters C1, C2, C3 and C4 binding sites among vertebrates. (H) Genomic location of clusters $\mathrm{C} 1$ to $\mathrm{C} 4$ binding sites identified by using the web-based CEAS tool. UTR, Untranslated region.

Similarity in the composition of binding sites for FOXM1, ERK2 and ER $\alpha$ and directionality of gene regulation marked classes of genes that are part of the same functional category, denoted as clusters $\mathrm{C} 1$ to $\mathrm{C} 4$ (Figure 2F). We further characterized these clusters based on GO enrichment, transcription factor prediction using SeqPos, genomic distribution and binding site conservation among species using CEAS. Cluster 1 (C1) was represented by binding sites occupied by FOXM1 and ERK2 and was enriched in genes involved in stem cell development, cellcycle $\mathrm{G}_{2}-\mathrm{M}$-related genes and transforming growth factor $\beta$, platelet-derived growth factor and hypoxia-inducible factor $2 \alpha$ (HIF2 $\alpha$ ) signaling pathways (Table 1). FOXM1 and GATA binding motifs were significantly enriched in this

Table 1 Enriched Gene Ontology functions, pathways and transcription factor motifs in the seqMINER-identified FOXM1 binding site clusters ${ }^{\mathrm{a}}$

\begin{tabular}{|c|c|c|}
\hline & Enriched GO functions and pathways & Enriched TFs \\
\hline \multirow[t]{4}{*}{ Cluster 1} & - Cell-cycle $\mathrm{G}_{2}-\mathrm{M}$ & FOXM1, GATA \\
\hline & - Stem cell development & \\
\hline & - Increased adenoma & \\
\hline & - TGF- $\beta$, PDGF and HIF2a signaling pathways & \\
\hline \multirow[t]{2}{*}{ Cluster 2} & - Genes regulated by ESR1 & ERa, FOXM1, CREB, ATF3 \\
\hline & - Genes upregulated in the luminal B subtype of breast cancer & \\
\hline \multirow[t]{13}{*}{ Cluster 3} & Subgroup A & \\
\hline & $\begin{array}{l}\text { - Genes associated with acquired endocrine therapy resistance } \\
\text { in breast tumors expressing ESR1 }\end{array}$ & ERa, FOXM1, AP-1 \\
\hline & - Focal adhesion & \\
\hline & • Neoplasm & \\
\hline & Subgroup B & FOXM1, ERa, AP-1 \\
\hline & - Cell substrate adherens junction & \\
\hline & - Cytoskeleton regulation and rearrangement & \\
\hline & - Neoplasm & \\
\hline & - Response to hypoxia & \\
\hline & Subgroup C & FOXM1, GATA, ERa \\
\hline & • Epithelial cell development & \\
\hline & • p53 pathway & \\
\hline & - HIF1a transcription factor network & \\
\hline \multirow[t]{4}{*}{ Cluster 4} & • Translation & FOXM1, GATA, Elk, AP-1, JunD \\
\hline & - Mammary gland hyperplasia & \\
\hline & - Abnormal apoptosis & \\
\hline & - Abnormal mitotic index & \\
\hline
\end{tabular}

${ }^{a}$ AP-1, Activator protein 1; ATF3, Activating transcription factor 3; CREB, cAMP response element-binding protein; ERa, Estrogen receptor a; GO, Gene Ontology; HIF, Hypoxia-inducible factor; PDGF, Platelet-derived growth factor; TFs, Transcription factors; TGF- $\beta$, Transforming growth factor $\beta$. 
cluster. Further, $\mathrm{C} 1$ and $\mathrm{C} 4$ had the highest sequence conservation among species (Figure 2G) and had a substantial presence of binding sites at proximal promoter genomic locations (Figure 2H, red), with clusters $\mathrm{C} 2$ and $\mathrm{C} 3$ showing the greatest proportion of binding sites at enhancer and intronic regions.

Cluster C2 (Figure 2F) was represented by binding sites containing ER $\alpha$, FOXM1 and ERK2. Genes harboring these binding sites were classified as ER $\alpha$ regulated genes that belonged to the luminal $\mathrm{B}$ breast cancer subtype (Table 1). These binding sites were mainly localized at enhancers (Figure $2 \mathrm{H}$ ) and were enriched for ER $\alpha$, FOXM1, CREB and ATF3 binding motifs. Cluster C3 was also characterized by occupancy by FOXM1 and ER $\alpha$, but not ERK2, and was associated with genes expressed in endocrine-resistant cells and genes associated with cytoskeletal regulation, focal adhesion, epithelial cell development, the p53 pathway and the HIF1 $\alpha$ network (Table 1). C4 binding sites were mainly enriched in genes involved in translation, mammary gland hyperplasia and abnormal apoptosis and mitotic index (Table 1). FOXM1, GATA, Elk, activator protein 1 and JunD motifs were enriched at these sites. In Additional file 2: Figure S1, we also present for comparison the binding site clustering pattern we obtained for ER $\alpha$ in MCF-7 cells after treatment with control vehicle, TAM or estradiol (E2) to explore if FOXM1 and ER $\alpha$ co-occupy chromatin sites in the presence of E2; this divided C3 into three subgroups
(Additional file 2: Figure S1). This analysis suggests that FOXM1 might act as a pioneering factor for ER $\alpha$ binding in the presence of TAM and E2. The cluster patterns indicate that FOXM1 has specific ( $\mathrm{C} 1$ and $\mathrm{C} 4)$ and common binding sites with ER $\alpha$ (C2 and $\mathrm{C} 3$ ) and highlight the ER $\alpha$-dependent and -independent roles of FOXM1 in the breast cancer phenotype.

We compared the FOXM1 binding sites from our study done in cells treated with $10^{-6} \mathrm{M}$ 4-OH-TAM with those described in the only other report on FOXM1 binding sites in MCF-7 cells, from Sanders et al. [14] (Additional file 2: Figure S2). In that study, these binding sites were examined in fetal bovine serum with no added hormone or hormone antagonist. Of the FOXM1 binding sites we identified in TAM-treated cells, 55\% were also found in the Sanders et al. study. Many FOXM1 chromatin binding sites differed, however, no doubt reflecting the very different cell treatment conditions.

\section{Cluster 1 genes can discriminate between patient breast tumors with different clinical outcomes}

Genes within $20 \mathrm{~kb}$ of binding sites belonging to cluster $\mathrm{C} 1$ and whose expression was impacted by FOXM1 knockdown were used to generate a gene predictor that was employed to interrogate two large, independent data sets $[38,41]$ of $E R \alpha$-positive breast cancer patients treated with TAM. Hierarchical clustering was used to stratify patients according to the expression of this $\mathrm{C} 1$ gene signature. As shown in Figures $3 \mathrm{~A}$ and $3 \mathrm{~B}$, the $\mathrm{C} 1$
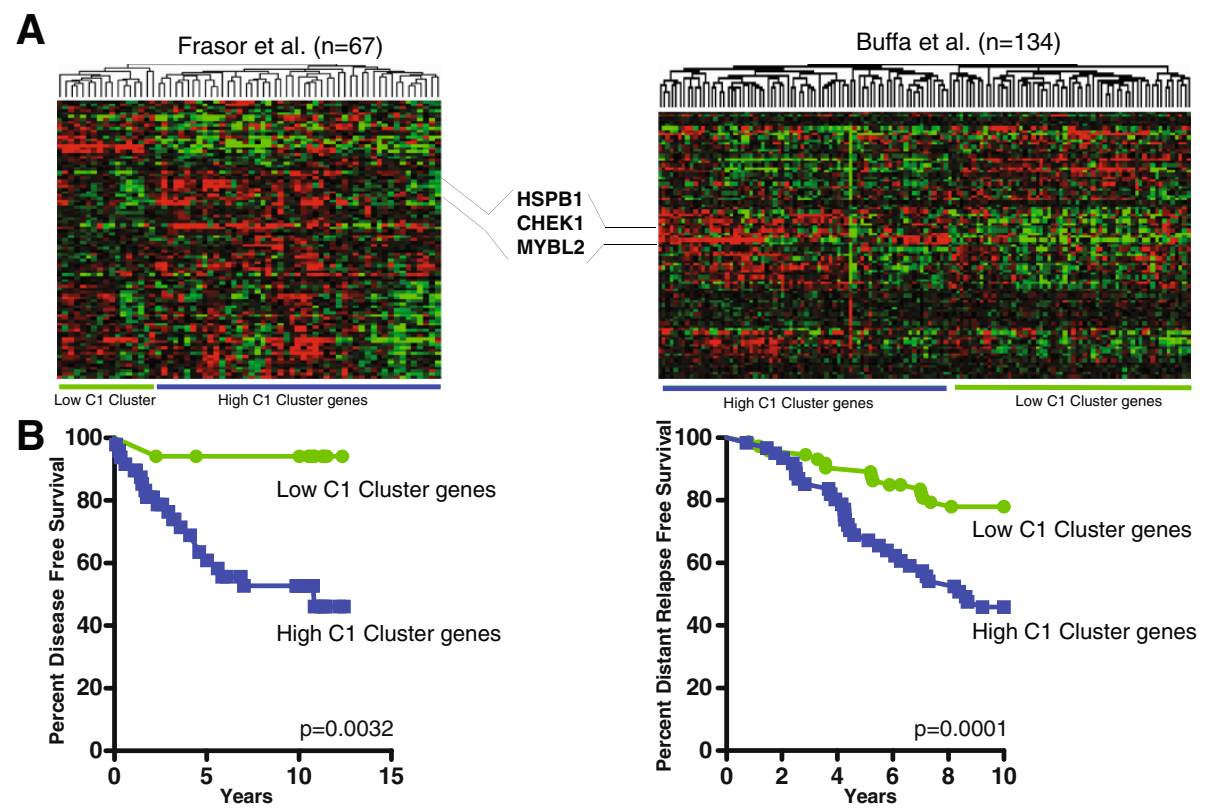

Figure 3 Expression of FOXM1-regulated genes in cluster C1 stratify breast cancer patients based on outcomes. (A) Heat map of hierarchical clustering of the expression of FOXM1-regulated C1 genes in two independent cohorts of patients treated with tamoxifen. Frasor et al. [38], Buffa et al. [41]. (B) Kaplan-Meier stratification of samples based on expression and hierarchical clustering of C1 genes. 
signature very effectively stratified the patients based on clinical outcome, indicating that FOXM1 regulatory sites and associated target genes may play a pivotal role in tumor progression and TAM resistance. Of interest, this signature included the genes HSPB1, CHEK1 and $M Y B L 2 / B-M Y B$, all of which have functions known to be associated with a poor prognosis [46-48].

\section{FOXM1 recruitment to $\mathrm{C} 1$ chromatin binding sites and impact of FOXM1 and extracellular signal-regulated kinase 2 inhibition}

To investigate the relationship between FOXM1 and ERK2 at sites of cobinding in cluster $\mathrm{C} 1$, we examined the recruitment of FOXM1 and ERK2 following the treatment of MCF-7 cells with 4-OH-TAM or vehicle in cells treated or not with a FOXM1-specific inhibitor (p19 ${ }^{\mathrm{ARF}} 26-44$ peptide) [49] or with a MEK1 inhibitor (AZD6244). We performed ChIP followed by qPCR for regions where both FOXM1 and ERK2 were identified in four of these genes: SIRT1, MYBL2, CHEK1 and ABCG2 (Figure 4A). By Western blot analysis, we show the effect of ERK1 and ERK2 knockdown on the levels of FOXM1 (Figure 4B) and the effect of FOXM1 inhibition by ARF on the levels of pMAPK (Figure 4C). Inhibition of FOXM1 by ARF or inhibition of ERK2 activation by MEK1 using a MEK1 inhibitor significantly decreased the recruitment of FOXM1 after 4-OH-TAM treatment to all four genes tested (Figure 4D). We also performed ChIP for ERK2 target genes before and after ARF treatment, and we observed a significant reduction in the recruitment of ERK2 to target gene loci after FOXM1 inhibitor treatment (Figure 4E). Moreover, the results of ChIP-reChIP experiments (Figure 4F) indicated that FOXM1 and ERK2 co-occupy the investigated genomic loci. We also modulated FOXM1 downward by inhibition (ARF) or by knockdown (siRNA) or upward by overexpression, and then examined effects on the mRNA levels (Figure 4G) of genes harboring these binding sites within a 20-kb window and on FOXM1 protein levels (Figure 4H). We observed that FOXM1 was crucial for the expression of SIRT1, MYBL2, CHEK1 and ASCG2 (Figure 4G). In addition, MEK1 inhibitor AZD6244 blocked TAM stimulation of these FOXM1 target genes (Figure 4I). These findings suggest that the binding of both FOXM1 and ERK2 to these chromatin binding sites is required for expression of these genes. Of note, transient knockdown of ERK1 or ERK2 significantly decreased the cellular level of FOXM1 and inhibition of FOXM1 also decreased the level of pMAPK, suggesting that they are part of an interdependent regulatory loop.

\section{FOXM1 transcription program drives expansion of cancer stem-like cells}

$\mathrm{GO}$ analysis of the $\mathrm{C} 1$ cluster revealed enrichment for stem cell-related genes such as $A B C G 2$ and SIRT1 and for the nuclear transcription factors NF-YA, NF-YB and $N F-Y C$. On the basis of our bioinformatics analyses, we therefore hypothesized that the FOXM1 transcription program might play a role in the expansion of the CSC population and that this phenomenon might promote the acquisition of endocrine resistance. To validate this hypothesis, we first investigated the percentage of CSCs by FACS using CD44 and CD24 markers in MCF-7 parental cells, in siFOXM1 and in FOXM1-overexpressing MCF-7 cells, and in TamR cells. Interestingly, we observed that TamR cells had a fivefold higher percentage of CSCs compared to parental MCF-7 cells (Figure 5A). Furthermore, cells overexpressing FOXM1 had a CSC enrichment of about eightfold compared to parental cells (Figure 5A) and increased levels of CD44 as shown by a FACS intensity plot (Figure $5 \mathrm{~B}$ ) and by a Western blot (Figure $5 \mathrm{C}$ ). We also monitored the level of CD44 mRNA during the development of endocrine resistance and observed that CD44 mRNA increased with weeks of 4-OH-TAM exposure (Figure 5D). Thus, elevated FOXM1 is associated with increased expression of CSC markers.

Given recent reports describing ABCG2 as a marker of CSCs [50-52], and on the basis of our gene expression data, we compared the levels of ABCG2 in MCF-7 parental and TamR cells after FACS separation and observed a great enrichment (approximately 50 -fold) in the ABCG2+ population in the TamR cells (Figure 5E). Moreover, ABCG2 mRNA levels increased progressively over time with TAM exposure, with the level increasing by about fivefold by 10 weeks and by approximately twelvefold by 100 weeks (Figure 5F). Next, to evaluate the functional role of FOXM1 in regulating the expression of ABCG2 and its involvement in TAM resistance, we reduced the level of ABCG2 by siRNA-mediated knockdown in TamR cells to $20 \%$ of the initial ABCG2 mRNA level (Additional file 2: Figure S4) and then tested cell sensitivity to 4-OH-TAM. As shown in Figure 5G, 4-OH-TAM stimulated proliferation of the control TamR cells, whereas cells rendered deficient in ABCG2 became sensitive to growth suppression by 4-OH-TAM (Figure 5G).

Next, by FACS, we separated TamR cells based on the ABCG2 expression marker (Figure $5 \mathrm{H}$ ) and evaluated cell proliferation in response to 4-OH-TAM in ABCG2+ and ABCG2- cells, with or without FOXM1 knockdown. Interestingly, 4-OH-TAM treatment in the control ABCG2+ cell population elicited growth stimulation, whereas FOXM1 knockdown in ABCG2+ cells prevented this growthstimulatory effect (Figure 5I). Thus, the ABCG2+ population appears to contribute to the endocrine-resistant phenotype (Figure 5I). Because the ABCG2+ and ABCG2- cell populations express ER $\alpha$ mRNA at levels similar to each other and to those of the overall TamR cell population (Figure 5J), changes in ER $\alpha$ level do not explain their differences in proliferative response to TAM. 


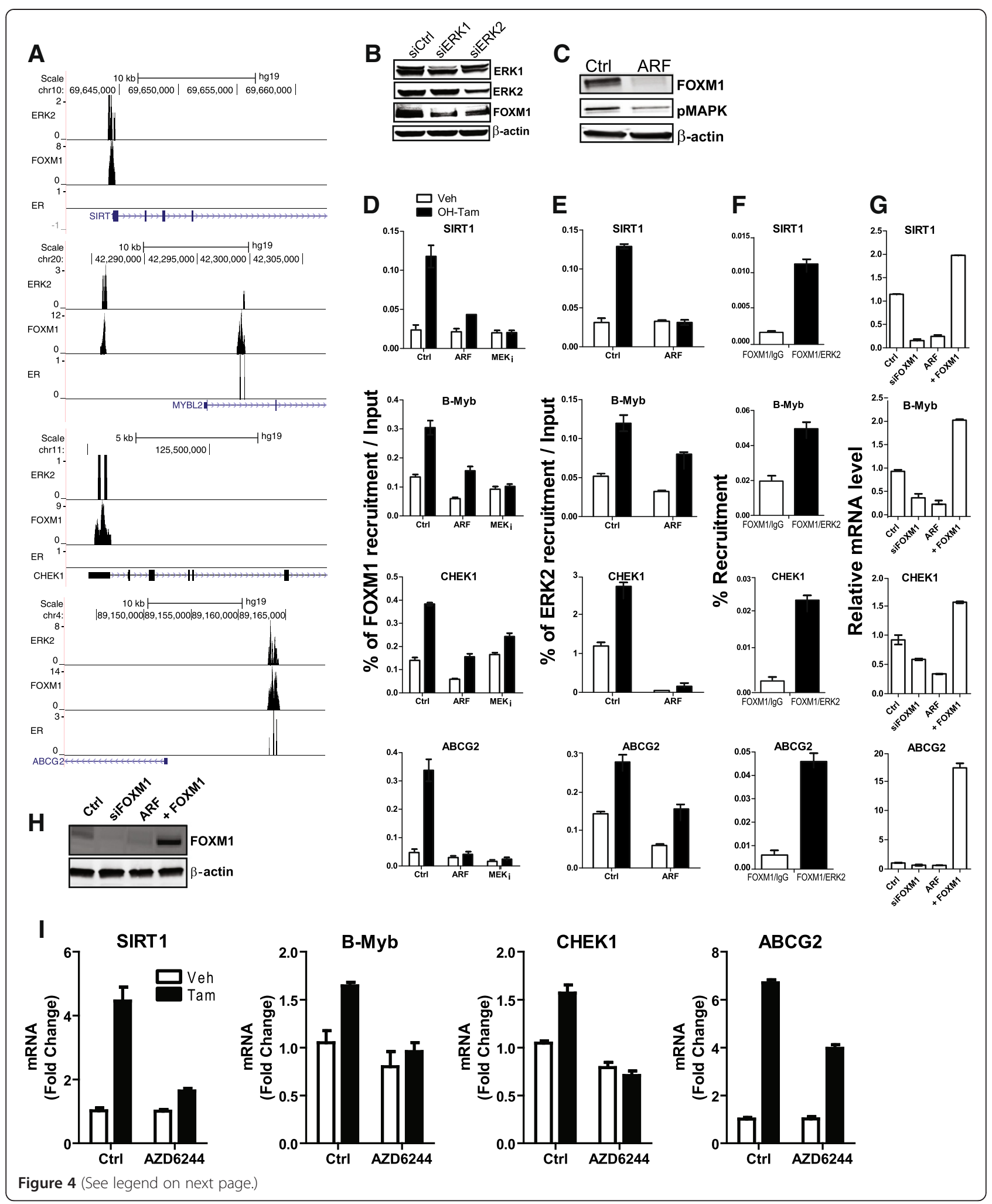


(See figure on previous page.)

Figure 4 FOXM1 and ERK2 co-occupy genomic loci of cluster C1 genes, and impact of knockdown of FOXM1, or ERK1, or ERK2, or treatment with FOXM1 inhibitor. (A) UCSC Browser location of the binding sites we identified for FOXM1, estrogen receptor a and extracellular signal-regulated kinase 2 (ERK2) for four representative genes in the C1 cluster (SIRT1, B-Myb, CHEK1 and ABCG2). (B) Western blot showing ERK1, ERK2 and FOXM1 levels after small interfering RNA (siRNA) knockdown of ERK1 or ERK2. (C) Western blot showing FOXM1 and phosphorylated mitogen-activated protein kinase levels after alternate reading frame (ARF) (FOXM1 inhibitor) treatment. (D) FOXM1 recruitment to chromatin sites was assessed after ARF or extracellular signal-regulated kinase kinase 1 (MEK1) inhibitor treatment and followed by quantitative RT-PCR (qRT-PCR). (E) Chromatin immunoprecipitation (ChIP) for ERK2 was assessed after ARF or control vehicle treatment. (F) ChIP-reChIP showing binding site CO-occupancy by FOXM1 and ERK2. Immunoprecipitation was done first for FOXM1 and then for ERK2. (G) Levels of representative C1 genes in siCtrl- or siFOXM1-treated cells or in cells with ARF treatment or overexpression of FOXM1. (H) Western blot showing FOXM1 levels after siRNA or ARF treatment or overexpression. (I) MEK1 inhibitor blocks tamoxifen (TAM) stimulation of FOXM1 target genes. Cells were pretreated with $10 \mu M$ MEK1 (AZD6244) or vehicle for 45 minutes and then treated with vehicle $(0.1 \% \mathrm{EtOH})$ or $1 \mu \mathrm{M}$ TAM in the presence or absence of inhibitor for 4 hours. RNA was isolated and $\mathrm{qPCR}$ analysis was done.

To examine the self-renewal properties of these cells, we sorted out ABCG2+ and ABCG2- TamR cells and cultured the different populations for 12 days. Because one of the defining characteristics of CSCs is their ability to transport Hoechst dye, which is attributable to expression of ABCG2, we examined Hoechst staining by FACS analysis. As shown in Figure 5K, ABCG2+ cells had a higher percentage of side population (SP) Hoechst-negative cells compared to the overall population of TamR cells or the ABCG2- cells.

Soft agar and three-dimensional Matrigel spheroid formation assays with the ABCG2+, ABCG2- and total TamR cell populations revealed that ABCG2+ cells not only had a more rapid growth rate compared to either TamR or ABCG2- cells (Figure 5L) but also formed more colonies (Figure 5M) and larger colonies (Figure 5N).

\section{FOXM1 overexpression increases breast cancer cell aggressiveness and the expression of markers of epithelial-to-mesenchymal transition and cytoskeletal rearrangement}

We observed a significant increase in the ABCG2+ population after overexpression of FOXM1 in MCF-7 cells for 72 hours, as examined by FACS (Figure 6A). Through evaluation of the functional role of FOXM1 in these cells by knockdown and overexpression, different phenotypes were clearly observed in three-dimensional Matrigel by as soon as 6 days (Figure 6B). No colony formation was seen in the FOXM1-knockdown (siFOXM1) population, whereas MCF-7 parental cells developed dense, round colonies. Cells overexpressing FOXM1 showed a very distinct phenotype characterized by branching chains of cells and cellular protrusions indicative of a migratory phenotype (Figure 6B). We also examined cell invasiveness using Boyden chambers and confirmed that FOXM1 overexpression engendered a more invasive phenotype, whereas invasion was decreased by FOXM1 reduction (Figure 6C).

The invasion process is associated with increases in EMT markers and loss of E-cadherin and requires cytoskeletal rearrangement. Hence, it was of interest that altering the level of FOXM1 modulated EMT markers SNAIL, TWIST, CXCR4 and E-cadherin (Figure 6D). Further, our binding site cluster analysis revealed an enrichment of FOXM1 and ER $\alpha$ co-occupancy on genes involved in cytoskeleton regulation and rearrangement. These include Rho-GTPase, CDC42 and RhoB. We observed that the expression of these genes was critically dependent on FOXM1 and was markedly altered by changing the level of FOXM1 (Figure 6E). We made similar observations in another ER+ cell line, T47Dnamely, an increase in TAM-regulated growth suppression and a decrease in $A B C G 2, C D C 42$ and $R h o B$ gene and protein expression, as well as in pMAPK level, after treatment of the cells with the FOXM1 inhibitor ARF (Additional file 2: Figure S3).

As seen in Figure 6F, when we compared the levels of FOXM1, CDC42 and RhoB in the sorted ABCG2+ and ABCG2- populations and in the total TamR cells, we consistently observed higher expression of FOXM1 and Rho-GTPase genes in ABCG2+ cells (Figure 6F). Consonant with this, ABCG2+ cells had higher invasion capability compared to ABCG2- cells or the total TamR population (Figure 5G). Hence, the subpopulation of ABCG2+ cells within the overall TamR cell population is responsible for the more invasive phenotype of the TamR cells.

\section{Discussion}

Our findings reveal that TAM resistance is associated with upregulation of FOXM1 and with a FOXM1dependent gene expression program that enhances cell proliferation and invasiveness and elicits an increase in the proportion of CSCs within the breast cancer cell population. These cells expressed many markers associated with stem cells and with decreased patient survival [26], including CD44+ and CD24-/low markers, and elevated EMT markers and properties. They also showed high expression of $\mathrm{ABC}$ transporters that can result in tumor stem-like cells being resistant to conventional 


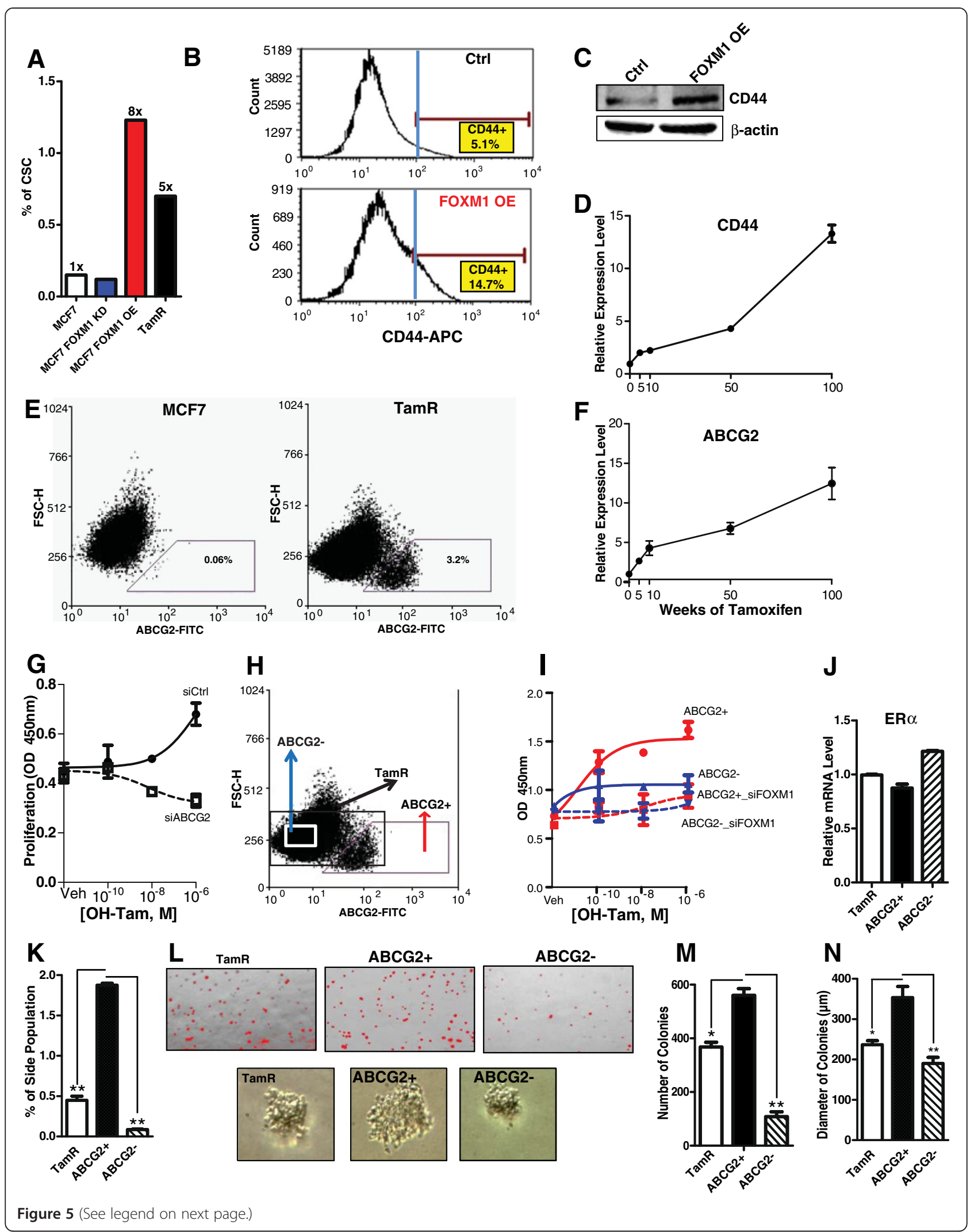




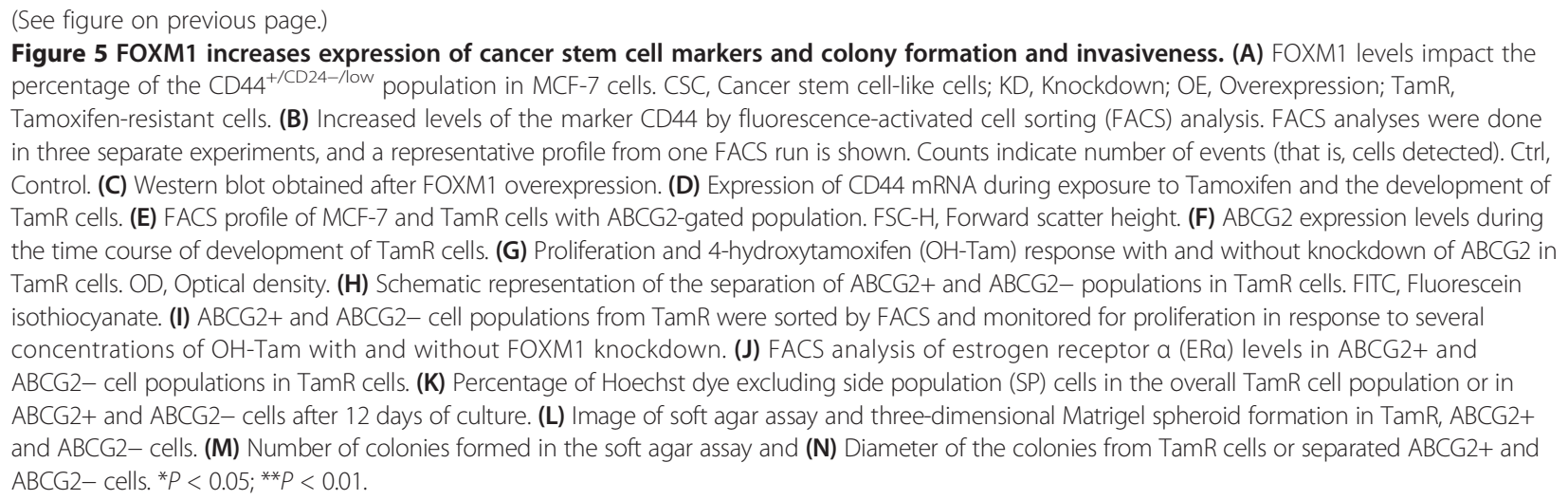

therapies due to drug efflux. These observations provide guidance for how one might optimally combine agents targeting specific characteristics of CSCs with conventional treatments that reduce tumor bulk, thereby effecting longterm benefits of ablating not only the overwhelming majority of the differentiated tumor cells but also removal of the more endocrine-resistant CSCs that can result in repopulation of the tumor [53-56]. Indeed, inherent drug resistance of CSCs is considered to be a crucial limitation to treatment effectiveness [56].

We found that the CSCs represent only a small proportion of the MCF-7 cell population, but that this fraction is increased fivefold in TamR cells. Our observations uncover a novel role for FOXM1 in inducing expansion of the CSClike population and in promoting an aggressive and endocrine-resistant phenotype. These effects of FOXM1 likely underlie the strong association we have observed between high tumor FOXM1 and poor clinical outcome for patients with ER+ breast cancers. Our examination of several large data sets cumulatively representing about 1,000 ER+ breast tumors indicates that high FOXM1 expression occurs in about $20 \%$ of ER+ breast cancers. Of note, the authors of a recent report showed that FOXM1 and its regulated target genes AURKA, AURKB and BIRC5/survivin display the greatest prognostic discrimination among a panel of genes analyzed for overall survival of patients with ER+ breast cancer and an intermediate Oncotype DX 21gene recurrence score. High expression of these genes predicts a poorer outcome and suggests more aggressive selection of adjuvant chemotherapy for these patients [57].

As schematized in the model (Figure $6 \mathrm{H}$ ), we show that FOXM1 is elevated by TAM in a time-dependent manner and that its expression is associated with markers of TAM resistance. In previous studies, we identified the association between FOXM1 and 14-3-3 to be upregulated by TAM and elevated in TAM-resistant tumors [13] via deregulation of miR-451 that targets 14-3$3 \zeta$ [42] (Figure 6H). Our data now reveal that FOXM1, a member of the family of forkhead transcription factors, fosters the enrichment of CSCs expressing stem cell markers (for example, ABCG2, NF-YA, NF-YB and NF$\mathrm{YC}$ ), mitosis-related genes and genes fostering invasiveness and motility (Rho-GTPases).

By ChIP-Seq and ChIP-reChIP, we show that TAM induced recruitment of FOXM1 to the promoter regions of cell-cycle mitosis-related genes and genes encoding stem cell markers in MCF-7 and TamR cells, supporting our hypothesis that FOXM1 promotes the expansion of a highly proliferative CSC-like progenitor population that is capable of self-renewal and can give rise to differentiated progeny. We also observed FOXM1 upregulation of EMT markers.

We focused much of this study on our novel finding of the regulation by FOXM1 of stem cell-related genes that were found by seqMINER analysis to be enriched in the $\mathrm{C} 1$ cluster. Moreover, this cluster was also enriched for targets of miR-34a, recently reported to be important in regulating the expression of self-renewal genes [58]. Interestingly, the FOXM1 C1 cluster binding sites are co-occupied by ERK2, suggesting a sophisticated mechanism by which FOXM1 and MAPK signaling may participate in the development of endocrine resistance. Indeed, resistance to endocrine therapies is known to be associated with enhanced signaling through MAPK $[1-3,5,8,45]$. We show in this study that the inhibition of MAPK activation with MEK1 inhibitor, or alteration of FOXM1 expression by the specific inhibitor ARF, impaired the recruitment of these factors to chromatin, indicating that these two factors control each other's binding to $\mathrm{C} 1$ genomic regions and that their copresence is essential for the activation of transcription of $\mathrm{C} 1$ genes. Of note, it has been shown that PMAPK induces phosphorylation of FOXM1, enabling its translocation to the nucleus and binding to genomic elements [59].

Further, our study reveals the interdependence of FOXM1 and MAPKs, with FOXM1 regulating the expression of MAPK and FOXM1-knockdown decreasing the level of MAPK. Of interest, the binding sites co-occupied by 


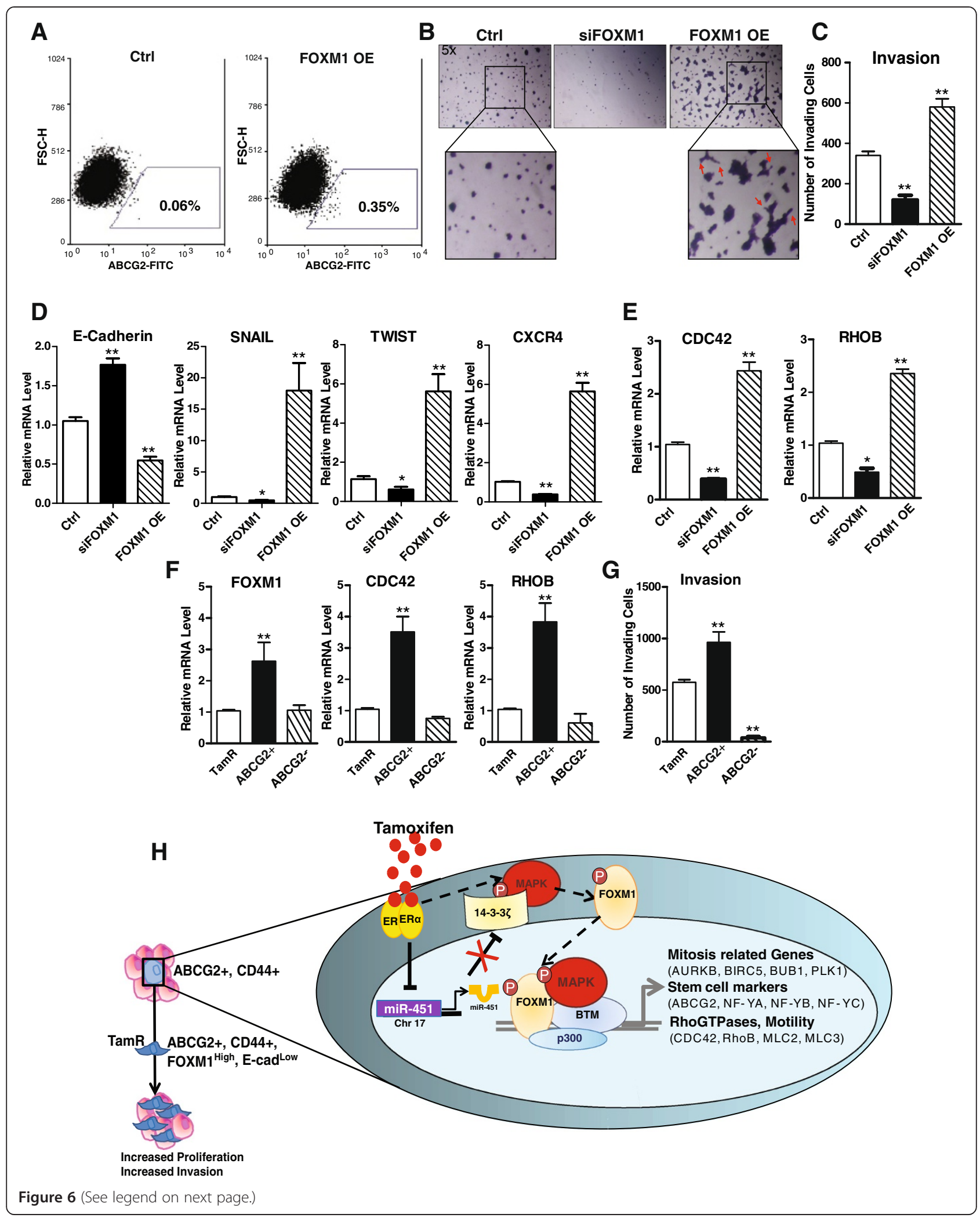




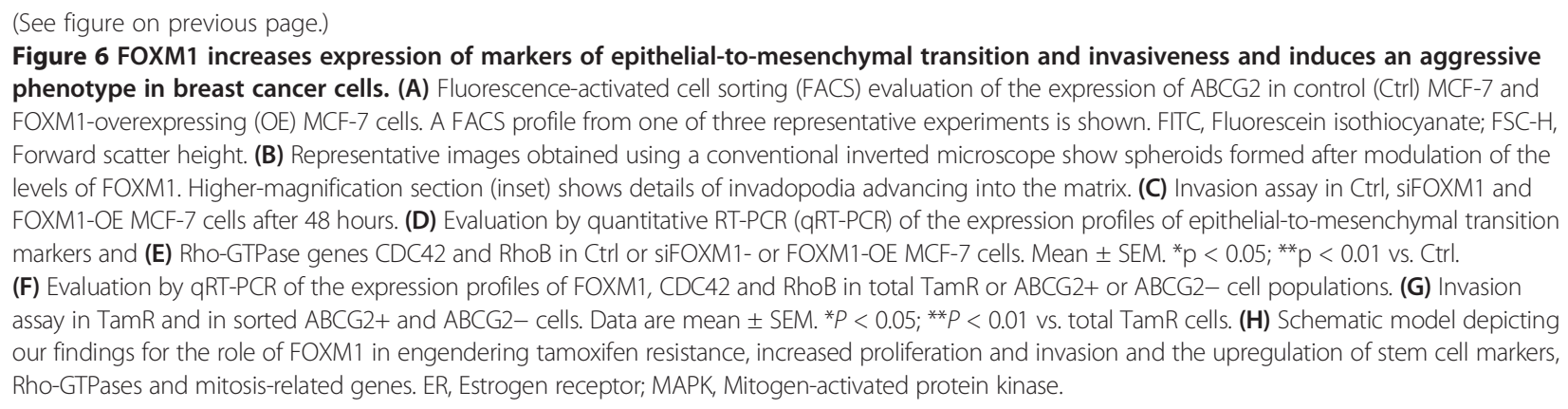

FOXM1 and MAPK are highly conserved among species, which suggests an evolutionarily conserved function for these genomic locations in different organisms. Moreover, our bioinformatics analysis of cluster C1 FOXM1-regulated genes was predictive of clinical outcome in women with TAMtreated tumors. Among these genes, we found well-described FOXM1 target genes such as B-Myb, c-Jun and c-Fos, as well as important genes involved in stem cell maintenance. Among the genes classified as CSC markers, we found multidrug resistance proteins ( $M D R 1, A B C G 5$ and $A B C G 2)$, the nuclear transcription factor $N F-Y A / B / C$ [60] and SIRT1 [61].

We concentrated in particular on studying the role of FOXM1 in regulating the expression of ABCG2 because ABCG2, also known as breast cancer resistance protein, belongs to the ATP-binding cassette family. A defining feature of CSCs is their ability to efflux Hoechst dye, leading to the identification of the SP that is associated with expression of the ABCG2 protein. Its expression has been found in several stem cell tissues, including lung and prostate cancer and glioblastoma [62,63]. Breast cancer SP cells have a high drug efflux capacity owing to functional expression of $\mathrm{ABC}$ transporters such as ABCG2. Although the mechanism by which multidrug resistance genes work in inducing chemotherapy resistance has been described previously, a recent study has implicated multidrug resistance proteins in hormone resistance by showing that ABCG2 can efflux TAM [64]. These reports support what we observed upon knockdown of either FOXM1 or ABCG2. With the reduction in cellular FOXM1 or ABCG2, or by inhibition of FOXM1 using ARF peptide, we were able to restore growth suppression by TAM to TamR cells, indicating that the levels of ABCG2 impact treatment response and that the upregulation of ABCG2 by FOXM1 could provide an explanation for the development of TAM therapy resistance.

In line with previous reports, our data show that our $\mathrm{ABCG} 2+\mathrm{SP}$ had higher invasiveness potential compared to ABCG2- cells upon examination by three-dimensional Matrigel culture and invasion assays. We further determined that this phenomenon is associated with their elevated expression of $C D C 42$ and $R h o B$ genes, which harbor FOXM1 binding sites co-occupied by ER $\alpha$.
Of note, we show that overexpression of FOXM1 induced a cell phenotype characterized by branching, extended chains of cells and cellular protrusions distinctive of a migratory phenotype and characterized by increased expression of $C D C 42$ and $R h o B$ and higher invasiveness. The GTP-binding proteins RhoB and CDC42 regulate the organization and turnover of the cytoskeleton and cell-matrix adhesions, which are a crucial feature in the acquisition of an invasive phenotype and the development of metastasis $[65,66]$. Further, in line with what has been previously reported, our data confirm the binding of FOXM1 to matrix metalloproteinases and VEGF [14] as well as the regulation of EMT markers, thereby associating FOXM1 at yet another level to the metastatic process [20].

\section{Conclusions}

Collectively, our findings define FOXM1 as a master regulator of Rho-GTPase and stem cell marker expression and imply that reducing FOXM1 expression might be effective in blocking tumor progression in several critical ways: by decreasing the expression of mitosis-related genes, by reducing invasion potential and by diminishing the proportion of CSCs, thereby enhancing sensitivity to cancer therapeutic agents. Indeed, the authors of several recent reports have shown FOXM1 to be associated with resistance to chemotherapeutic agents [67] and resistance to radiation treatment [68].

Moreover, our functional work clearly shows that rendering the FOXM1 pathway inactive by RNAi knockdown or by use of the p19 ARF $26-44$ peptide [49], a selective FOXM1 peptide inhibitor called ARF, was highly effective in restoring endocrine sensitivity and suppressing breast cancer aggressiveness. This ARF inhibitor has already been shown to be effective in suppressing the development of hepatocellular carcinoma in a preclinical model [69]. Taken together, our findings have clinical implications for breast cancer and potentially many other cancers where FOXM1/ pMAPK signaling pathways are active, and make a case for the use of FOXM1 inhibitors in combination with current therapies, including protein kinase inhibitors, to improve effectiveness and long-term patient response to treatments. 


\section{Additional files}

\section{Additional file 1: BED file detailing the FOXM1 and ERK2 binding regions.}

Additional file 2: Supplementary figures and figure legends.

\section{Abbreviations}

EMT: Epithelial-to-mesenchymal transition; ERK2: Extracellular signal-regulated kinase 2; ERa: Estrogen receptor a; PBS: Phosphate-buffered saline; siRNA: Small interfering RNA; TMA: Tissue microarray.

\section{Competing interests}

The authors declare that they have no competing interests.

\section{Authors' contributions}

$A B$ conceived of and designed the project; performed the gene expression, genomic and cell biological assays; analyzed the assay data and clinical patient data sets; and wrote the manuscript. ZME conceived of and designed the project; performed the gene expression, genomic and cell biological assays; analyzed the assay data and clinical patient data sets; and contributed to the writing of the manuscript. YJK carried out the $\mathrm{HCC}$ experiments and analyses on large clinical TMAs, analyzed the data and contributed to data interpretation and the writing of the manuscript. YLC carried out the IHC experiments and analyses on large clinical TMAs, analyzed the data and contributed to data interpretation and the writing of the manuscript. HL assisted in designing the project, performed gene expression and cell biological assays and analyzed these data, and contributed to the writing of the manuscript. BSK conceived of, designed and directed the project; oversaw the experiments; analyzed all data; and wrote the manuscript. All authors read and approved the final manuscript.

\section{Acknowledgments}

This research was supported by a grant from The Breast Cancer Research Foundation (to BSK).

\section{Author details}

'Department of Molecular and Integrative Physiology, University of Illinois at Urbana-Champaign, 524 Burrill Hall, Urbana, IL 61801, USA. ${ }^{2}$ Laboratory of Cancer Genomics and Molecular Pathology, Samsung Biomedical Research Institute, Samsung Medical Center, Irwon-dong Gangnam-gu, Seoul 135-710, Korea. ${ }^{3}$ Department of Pathology, Samsung Medical Center, Sungkyunkwan University School of Medicine, Irwon-ro 81, Gangnam-gu, Seoul 135-710, Korea. ${ }^{4}$ Department of Breast Cancer, Affiliated Tumor Hospital of Harbin Medical University, No.150 Haping Road, Nangag District, Harbin 150040, China.

Received: 18 February 2014 Accepted: 28 August 2014

Published online: 12 September 2014

\section{References}

1. Creighton CJ, Massarweh S, Huang S, Tsimelzon A, Hilsenbeck SG, Osborne CK, Shou J, Malorni L, Schiff R: Development of resistance to targeted therapies transforms the clinically associated molecular profile subtype of breast tumor xenografts. Cancer Res 2008, 68:7493-7501.

2. Schiff R, Massarweh S, Shou J, Osborne CK: Breast cancer endocrine resistance: how growth factor signaling and estrogen receptor coregulators modulate response. Clin Cancer Res 2003, 9:447S-454S.

3. Sengupta S, Schiff R, Katzenellenbogen BS: Post-transcriptional regulation of chemokine receptor CXCR4 by estrogen in HER2 overexpressing, estrogen receptor-positive breast cancer cells. Breast Cancer Res Treat 2009, 117:243-251

4. Shou J, Massarweh S, Osborne CK, Wakeling AE, Ali S, Weiss H, Schiff R: Mechanisms of tamoxifen resistance: increased estrogen receptor-HER2/ neu cross-talk in ER/HER2-positive breast cancer. J Natl Cancer Inst 2004, 96:926-935.

5. Arpino G, Wiechmann L, Osborne CK, Schiff R: Crosstalk between the estrogen receptor and the HER tyrosine kinase receptor family: molecular mechanism and clinical implications for endocrine therapy resistance. Endocr Rev 2008, 29:217-233.
6. Creighton CJ, Hilger AM, Murthy S, Rae JM, Chinnaiyan AM, El-Ashry D: Activation of mitogen-activated protein kinase in estrogen receptor a-positive breast cancer cells in vitro induces an in vivo molecular phenotype of estrogen receptor a-negative human breast tumors. Cancer Res 2006, 66:3903-3911.

7. Green KA, Carroll JS: Oestrogen-receptor-mediated transcription and the influence of co-factors and chromatin state. Nat Rev Cancer 2007, 7:713-722.

8. Massarweh S, Osborne CK, Creighton CJ, Qin L, Tsimelzon A, Huang S, Weiss H, Rimawi M, Schiff R: Tamoxifen resistance in breast tumors is driven by growth factor receptor signaling with repression of classic estrogen receptor genomic function. Cancer Res 2008, 68:826-833.

9. O'Brien CS, Howell SJ, Farnie G, Clarke RB: Resistance to endocrine therapy: Are breast cancer stem cells the culprits? J Mammary Gland Biol Neoplasia 2009, 14:45-54.

10. O'Brien CA, Kreso A, Dick JE: Cancer stem cells in solid tumors: an overview. Semin Radiat Oncol 2009, 19:71-77.

11. Jordan CT: Cancer stem cells: controversial or just misunderstood? Cell Stem Cell 2009, 4:203-205.

12. Bergamaschi A, Frasor J, Borgen K, Stanculescu A, Johnson P, Rowland K, Wiley EL, Katzenellenbogen BS: $14-3-3 \zeta$ as a predictor of early time to recurrence and distant metastasis in hormone receptor-positive and -negative breast cancers. Breast Cancer Res Treat 2013, 137:689-696.

13. Bergamaschi A, Christensen BL, Katzenellenbogen BS: Reversal of endocrine resistance in breast cancer: interrelationships among 14-3-3ろ, FOXM1, and a gene signature associated with mitosis. Breast Cancer Res 2011, 13:R70.

14. Sanders DA, Ross-Innes CS, Beraldi D, Carroll JS, Balasubramanian S: Genome-wide mapping of FOXM1 binding reveals co binding with estrogen receptor a in breast cancer cells. Genome Bio/ 2013, 14:R6.

15. Wonsey DR, Follettie MT: Loss of the forkhead transcription factor FoxM1 causes centrosome amplification and mitotic catastrophe. Cancer Res 2005, 65:5181-5189.

16. Chen PM, Wu TC, Shieh SH, Wu YH, Li MC, Sheu GT, Cheng YW, Chen CY, Lee $H$ : MnSOD promotes tumor invasion via upregulation of FoxM1-MMP2 axis and related with poor survival and relapse in lung adenocarcinomas. Mol Cancer Re 2013, 11:261-271.

17. Chu XY, Zhu ZM, Chen LB, Wang JH, Su QS, Yang JR, Lin Y, Xue LJ, Liu XB, Mo XB: FOXM1 expression correlates with tumor invasion and a poor prognosis of colorectal cancer. Acta Histochem 2012, 114:755-762.

18. Wang Z, Park HJ, Carr JR, Chen YJ, Zheng Y, Li J, Tyner AL, Costa RH, Bagchi S, Raychaudhuri P: FoxM1 in tumorigenicity of the neuroblastoma cells and renewal of the neural progenitors. Cancer Res 2011, 71:4292-4302.

19. He SY, Shen HW, Xu L, Zhao XH, Yuan L, Niu G, You ZS, Yao SZ: FOXM1 promotes tumor cell invasion and correlates with poor prognosis in early-stage cervical cancer. Gynecol Oncol 2012, 127:601-610.

20. Raychaudhuri P, Park HJ: FoxM1: a master regulator of tumor metastasis. Cancer Res 2011, 71:4329-4333.

21. Wang $Y$, Wen L, Zhao SH, Ai ZH, Guo JZ, Liu WC: FoxM1 expression is significantly associated with cisplatin-based chemotherapy resistance and poor prognosis in advanced non-small cell lung cancer patients. Lung Cancer 2013, 79:173-179.

22. Xia JT, Wang $H$, Liang $L$, Peng BG, Wu ZF, Chen LZ, Xue L, Li Z, Li W: Overexpression of FOXM1 is associated with poor prognosis and clinicopathologic stage of pancreatic ductal adenocarcinoma. Pancreas 2012, 41:629-635.

23. Carr JR, Kiefer MM, Park HJ, Li J, Wang Z, Fontanarosa J, DeWaal D, Kopanja D, Benevolenskaya EV, Guzman G, Raychaudhuri P: FoxM1 regulates mammary luminal cell fate. Cell Rep 2012, 1:715-729.

24. Zhang Y, Zhang N, Dai B, Liu M, Sawaya R, Xie K, Huang S: FoxM1B transcriptionally regulates vascular endothelial growth factor expression and promotes the angiogenesis and growth of glioma cells. Cancer Res 2008, 68:8733-8742.

25. Herman ME, Katzenellenbogen BS: Response-specific antiestrogen resistance in a newly characterized MCF-7 human breast cancer cell line resulting from long-term exposure to trans-hydroxytamoxifen. J Steroid Biochem Mol Biol 1996, 59:121-134.

26. Shipitsin M, Campbell LL, Argani P, Weremowicz S, Bloushtain-Qimron N Yao J, Nikolskaya T, Serebryiskaya T, Beroukhim R, Hu M, Halushka MK, Sukumar S, Parker LM, Anderson KS, Harris LN, Garber JE, Richardson AL, Schnitt SJ, Nikolsky Y, Gelman RS, Polyak K: Molecular definition of breast tumor heterogeneity. Cancer Cell 2007, 11:259-273.

27. Madak-Erdogan Z, Ventrella R, Petry L, Katzenellenbogen BS: Novel roles for ERK5 and cofilin as critical mediators linking ERa-driven transcription, 
actin reorganization, and invasiveness in breast cancer. Mol Cancer Res 2014, 12:714-727.

28. Madak-Erdogan Z, Charn TH, Jiang Y, Liu ET, Katzenellenbogen JA, Katzenellenbogen BS: Integrative genomics of gene and metabolic regulation by estrogen receptors $\alpha$ and $\beta$, and their coregulators. Mol Syst Biol 2013, 9:676.

29. Langmead B, Salzberg SL: Fast gapped-read alignment with Bowtie 2 . Nat Methods 2012, 9:357-359.

30. Zhang Y, Liu T, Meyer CA, Eeckhoute J, Johnson DS, Bernstein BE, Nusbaum C, Myers RM, Brown M, Li W, Liu XS: Model-based analysis of ChIP-Seq (MACS). Genome Biol 2008, 9:R137.

31. Hurtado A, Holmes KA, Ross-Innes CS, Schmidt D, Carroll JS: FOXA1 is a key determinant of estrogen receptor function and endocrine response. Nat Genet 2011, 43:27-33.

32. Ye T, Krebs AR, Choukrallah MA, Keime C, Plewniak F, Davidson I, Tora L: seqMINER: an integrated ChIP-seq data interpretation platform. Nucleic Acids Res 2011, 39:e35.

33. Liu T, Ortiz JA, Taing L, Meyer CA, Lee B, Zhang Y, Shin H, Wong SS, Ma J, Lei Y, Pape UJ, Poidinger M, Chen Y, Yeung K, Brown M, Turpaz Y, Liu XS: Cistrome: an integrative platform for transcriptional regulation studies. Genome Biol 2011, 12:R83.

34. Dennis G Jr, Sherman BT, Hosack DA, Yang J, Gao W, Lane HC, Lempicki RA: DAVID: database for annotation, visualization, and integrated discovery. Genome Biol 2003, 4:P3.

35. Huang DW, Sherman BT, Lempicki RA: Systematic and integrative analysis of large gene lists using DAVID bioinformatics resources. Nat Protoc 2009, 4:44-57.

36. McLean CY, Bristor D, Hiller M, Clarke SL, Schaar BT, Lowe CB, Wenger AM, Bejerano G: GREAT improves functional interpretation of cis-regulatory regions. Nat Biotechnol 2010, 28:495-501.

37. Galaxy/Cistrome data analysis. [http://cistrome.org/ap/]

38. Frasor J, Chang EC, Komm B, Lin CY, Vega VB, Liu ET, Miller LD, Smeds J, Bergh J, Katzenellenbogen BS: Gene expression preferentially regulated by tamoxifen in breast cancer cells and correlations with clinical outcome. Cancer Res 2006, 66:7334-7340.

39. PrimerBank: PCR Primers for Gene Expression Detection and Quantification. [http://pga.mgh.harvard.edu/primerbank/] (accessed 19 September 2014).

40. Hochberg Y, Benjamini Y: More powerful procedures for multiple significance testing. Stat Med 1990, 9:811-818.

41. Buffa FM, Camps C, Winchester L, Snell CE, Gee HE, Sheldon H, Taylor M, Harris AL, Ragoussis J: microRNA-associated progression pathways and potential therapeutic targets identified by integrated mRNA and microRNA expression profiling in breast cancer. Cancer Res 2011 71:5635-5645

42. Bergamaschi A, Katzenellenbogen BS: Tamoxifen downregulation of miR-451 increases 14-3-3 3 and promotes breast cancer cell survival and endocrine resistance. Oncogene 2012, 31:39-47.

43. Choi YL, Oh E, Park S, Kim Y, Park YH, Song K, Cho EY, Hong YC, Choi JS, Lee JE, Kim JH, Nam SJ, Im YH, Yang JH, Shin YK: Triple-negative, basal-like, and quintuple-negative breast cancers: better prediction model for survival. BMC Cancer 2010, 10:507. A published erratum appears in BMC Cancer 2011, 11:13.

44. Kwon MJ, Park S, Choi JY, Oh E, Kim YJ, Park YH, Cho EY, Nam SJ, Im YH, Shin YK, Choi YL: Clinical significance of CD151 overexpression in subtypes of invasive breast cancer. Br J Cancer 2012, 106:923-930.

45. Madak-Erdogan Z, Lupien M, Stossi F, Brown M, Katzenellenbogen BS: Genomic collaboration of estrogen receptor $a$ and extracellular signal-regulated kinase 2 in regulating gene and proliferation programs. Mol Cell Biol 2011, 31:226-236.

46. Gibert B, Eckel B, Gonin V, Goldschneider D, Fombonne J, Deux B, Mehlen P, Arrigo AP, Clezardin P, Diaz-Latoud C: Targeting heat shock protein 27 (HspB1) interferes with bone metastasis and tumour formation in vivo. Br J Cancer 2012, 107:63-70.

47. Sinha S, Singh RK, Bhattacharya N, Mukherjee N, Ghosh S, Alam N, Roy A, Roychoudhury S, Panda CK: Frequent alterations of LOH11CR2A, PIG8 and CHEK1 genes at chromosomal 11q24.1-24.2 region in breast carcinoma: clinical and prognostic implications. Mol Oncol 2011, 5:454-464.

48. Thorner AR, Hoadley KA, Parker JS, Winkel S, Millikan RC, Perou CM: In vitro and in vivo analysis of $B-M y b$ in basal-like breast cancer. Oncogene 2009 28:742-751.
49. Kalinichenko W, Major ML, Wang X, Petrovic V, Kuechle J, Yoder HM, Dennewitz MB, Shin B, Datta A, Raychaudhuri P, Costa RH: Foxm1b transcription factor is essential for development of hepatocellular carcinomas and is negatively regulated by the p19ARF tumor suppressor. Genes Dev 2004, 18:830-850.

50. Ding $X-w$ W, Wu J, Jiang C: ABCG2: a potential marker of stem cells and novel target in stem cell and cancer therapy. Life Sci 2010, 86:631-637.

51. Medema JP: Cancer stem cells: the challenges ahead. Nat Cell Biol 2013, 15:338-344

52. Zhang G, Wang Z, Luo W, Jiao H, Wu J, Jiang C: Expression of potential cancer stem cell marker ABCG2 is associated with malignant behaviors of hepatocellular carcinoma. Gastroenterol Res Pract 2013, 2013:782581.

53. Ginestier C, Hur MH, Charafe-Jauffret E, Monville F, Dutcher J, Brown M, Jacquemier J, Viens P, Kleer CG, Liu S, Schott A, Hayes D, Birnbaum D, Wicha MS, Dontu G: ALDH1 is a marker of normal and malignant human mammary stem cells and a predictor of poor clinical outcome. Cell Stem Cell 2007, 1:555-567.

54. Ischenko I, Seeliger H, Schaffer M, Jauch KW, Bruns CJ: Cancer stem cells: how can we target them? Curr Med Chem 2008, 15:3171-3184.

55. Visvader JE, Lindeman GJ: Cancer stem cells in solid tumours: accumulating evidence and unresolved questions. Nat Rev Cancer 2008, 8:755-768.

56. Zhou BB, Zhang H, Damelin M, Geles KG, Grindley JC, Dirks PB: Tumourinitiating cells: challenges and opportunities for anticancer drug discovery. Nat Rev Drug Discov 2009, 8:806-823.

57. Ahn SG, Lee HM, Lee HW, Lee SA, Lee SR, Leem SH, Jeong J, Chu IS: Prognostic discrimination using a 70-gene signature among patients with estrogen receptor-positive breast cancer and an intermediate 21-gene recurrence score. Int J Mol Sci 2013, 14:23685-23699.

58. Kashat M, Azzouz L, Sarkar SH, Kong D, Li Y, Sarkar FH: Inactivation of AR and Notch-1 signaling by miR-34a attenuates prostate cancer aggressiveness. Am J Trans/ Res 2012, 4:432-442

59. Ma RYM, Tong THK, Leung WY, Yao KM: Raf/MEK/MAPK signaling stimulates the nuclear translocation and transactivating activity of FOXM1. Methods Mol Biol 2010, 647:113-123.

60. Zhu J, Zhang Y, Joe GJ, Pompetti R, Emerson SG: NF-Ya activates multiple hematopoietic stem cell (HSC) regulatory genes and promotes HSC self-renewal. Proc Natl Acad Sci U S A 2005, 102:11728-11733.

61. Calvanese V, Lara E, Suárez-Alvarez B, Abu Dawud R, Vázquez-Chantada M, Martínez-Chantar ML, Embade N, López-Nieva P, Horrillo A, Hmadcha A, Soria B, Piazzolla D, Herranz D, Serrano M, Mato JM, Andrews PW, LópezLarrea C, Esteller M, Fraga MF: Sirtuin 1 regulation of developmental genes during differentiation of stem cells. Proc Natl Acad Sci U S A 2010, 107:13736-13741.

62. Tsunoda S, Okumura T, Ito T, Kondo K, Ortiz C, Tanaka E, Watanabe G, Itami A, Sakai $Y$, Shimada $Y$ : ABCG2 expression is an independent unfavorable prognostic factor in esophageal squamous cell carcinoma. Oncology 2006, 71:251-258.

63. Jin Y, Bin ZQ, Qiang H, Liang C, Hua C, Jun D, Dong WA, Qing L: ABCG2 is related with the grade of glioma and resistance to mitoxantone, a chemotherapeutic drug for glioma. J Cancer Res Clin Oncol 2009, 135:1369-1376.

64. Selever J, Gu G, Lewis MT, Beyer A, Herynk MH, Covington KR, Tsimelzon A, Dontu G, Provost P, Di Pietro A, Boumendjel A, Albain K, Miele L, Weiss H, Barone I, Ando S, Fuqua SA: Dicer-mediated upregulation of BCRP confers tamoxifen resistance in human breast cancer cells. Clin Cancer Res 2011, 17:6510-6521

65. Hall A: Rho GTPases and the actin cytoskeleton. Science 1998, 279:509-514.

66. Etienne-Manneville S, Hall A: Rho GTPases in cell biology. Nature 2002, 420:629-635.

67. Carr JR, Park HJ, Wang Z, Kiefer MM, Raychaudhuri P: FoxM1 mediates resistance to herceptin and paclitaxel. Cancer Res 2010, 70:5054-5063.

68. Kambach DM, Sodi VL, Lelkes PI, Azizkhan-Clifford J, Reginato MJ: ErbB2, FoxM1 and 14-3-3 3 prime breast cancer cells for invasion in response to ionizing radiation. Oncogene 2013, 33:589-598.

69. Gusarova GA, Wang IC, Major ML, Kalinichenko W, Ackerson T, Petrovic V, Costa $\mathrm{RH}$ : A cell-penetrating ARF peptide inhibitor of FoxM1 in mouse hepatocellular carcinoma treatment. J Clin Invest 2007, 117:99-111.

\section{doi:10.1186/s13058-014-0436-4}

Cite this article as: Bergamaschi et al.: The forkhead transcription factor FOXM1 promotes endocrine resistance and invasiveness in estrogen receptor-positive breast cancer by expansion of stem-like cancer cells. Breast Cancer Research 2014 16:436 